\title{
Metagenomic Analysis Reveals the Mechanism for the Observed Increase in Antibacterial Activity of Penicillin against Uncultured Bacteria Candidatus Liberibacter asiaticus Relative to Oxytetracycline in Planta
}

\author{
Chuanyu Yang ${ }^{1,2, *,+}$, Hanqing Hu ${ }^{1,+}$, Yihong $\mathrm{Wu}^{1}{ }^{1}$, Xiongjie Lin ${ }^{1}$, Goucheng Fan ${ }^{1}$, \\ Yongping Duan ${ }^{3}$, Charles Powell ${ }^{4}$, Veronica Ancona ${ }^{2}$ (D) and Muqing Zhang 4,5,* \\ 1 Institute of Fruit Tree Research, Fujian Academy of Agricultural Sciences, Fuzhou 350003, China; \\ hanqinghu@126.com (H.H.); redone233@163.com (Y.W.); linxj_019@163.com (X.L.); \\ guochengfan@126.com (G.F.) \\ 2 Citrus Center, Texas A\&M University-Kingsville, 312 N. International Blvd, Weslaco, TX 785799, USA; \\ Veronica.Ancona-Contreras@tamuk.edu \\ 3 USHRL-USDA-ARS, 2011 S. Rock Rd, Fort Pierce, FL 34945, USA; yongping.duan@ars.usda.gov \\ 4 IRREC-IFAS, University of Florida, 2199 S. Rock Rd, Fort Pierce, FL 34945, USA; capowell@ufl.edu \\ 5 State Key Lab for Conservation and Utilization of Subtropical Agri-Biological Resources, Guangxi University, \\ Nanning 530005, China \\ * Correspondence: Chuanyu.Yang@tamuk.edu (C.Y.); mqzhang@ufl.edu (M.Z.) \\ + These authors have contributed equally to this work.
}

\begin{abstract}
Citrus huanglongbing (HLB) is a devastating disease for the citrus industry. The previous studies demonstrated that oxytetracycline and penicillin are effective antibiotics against Candidatus Liberibacter asiaticus (CLas). However, since CLas is uncultured, the mechanisms of action of antibiotics against $C$ Las are still unclear. It was recently reported that the endophytic microbial communities are associated with the progression of citrus HLB after oxytetracycline and penicillin treatment. Therefore, we hypothesize that penicillin has greater antibacterial activity against CLas than oxytetracycline, which may be associated with the alteration of the structure and function of endophytic microbial communities in HLB-affected citrus in response to these antibiotics. To test this hypothesis, the microbiome of HLB-affected citrus leaves treated with these two antibiotics was analyzed using a metagenomic method. Our results indicate that the microbial structure and function in HLB-affected citrus were altered by these two antibiotics. The relative abundance of beneficial bacterial species, including Streptomyces avermitilis and Bradyrhizobium, was higher in penicillin-treated plants compared to those treated with oxytetracycline, and the relative abundance of the bacterial species (such as Propionibacterium acnes and Synechocystis sp PCC 6803) associated with CLas survival was lower for penicillin-treated plants compared to oxytetracycline-treated plants. These results indicate that penicillin has greater antibacterial activity against $C$ Las. Based on the metagenomic analysis, this study elucidated the mechanism for the observed increase in antibacterial activity of penicillin against $C$ Las. The data presented here are not only invaluable for developing eco-friendly and effective biocontrol strategies to combat citrus HLB, but also provide a method for revealing mechanism of antimicrobial against uncultured bacteria in host.
\end{abstract}

Keywords: citrus Huanglongbing; endophytic microbiome; oxytetracyline; penicillin; mode of action 


\section{Introduction}

Citrus huanglongbing (HLB) is one of the most devastating diseases of citrus. HLB spreads across most citrus-growing areas worldwide and has caused significant losses or declines in both production and profit of the citrus industry [1,2]. Three species of fastidious, phloem-restricted $\alpha$-proteobacteria: "Candidatus Liberibacter asiaticus" (CLas), "Candidatus Liberibacter americanus"(CLam), and "Candidatus Liberibacter africanus" (CLaf), which are transmitted by the psyllids Diaphorina citri or Trioza erytreae, are the major causative agents of HLB [1,2]. Of these three species, CLas is the most prevalent and the only species found in China and the United States. To date, there are no effective treatment strategies for infected trees in field, and there are no resistant commercial citrus varieties [3]. Chemotherapy has shown considerable promise for control of HLB in the short term.

The previous studies demonstrated that oxytetracycline and penicillin are effective antibiotics against $C$ Las $[4,5]$. Oxytetracycline are short-acting antibiotics that inhibit bacterial growth by inhibiting translation. It passively diffuses through porin channels in the bacterial membrane, binds reversible to the bacterial 30S ribosomal subunit and prevents the aminoacyl tRNA from binding to the A site of the ribosome [6]. Oxytetracycline is only bacteriostatic, application of this antibiotic against CLas was later discontinued and requiring treatment needed to be repeated each year [7]. Penicillin as Beta-lactam antibiotic, inhibit the growth of sensitive bacteria by inactivating enzymes located in the bacterial cell membrane, known as penicillin binding proteins, which are involved in cell wall synthesis [8]. Penicillin are considered to be bactericidal [9]. HLB-affected citrus treated with penicillin in greenhouse, displayed no HLB-symptom and no CLas was detected in the plants [10]. However, since CLas is uncultured, the modes of action of penicillin and oxytetracycline against CLas are still unclear.

The plant microbiome in the rhizosphere, phyllosphere, and endosphere plays a key role in plant growth and health [11]. The structure and function of endophyte microbial communities in HLB-affected citrus are also altered by oxytetracycline and penicillin. The CLas strain Ishi-1 is reported not to be susceptible to oxytetracycline in vitro, but oxytetracycline treatment did eliminate a particular sub-community dominated by the families Comamonadaceae, Flavobacteriaceae, Microbacteriaceae, and Pseudomonadaceae to decrease CLas survival [12]. Several studies also indicated that when penicillin was applied to HLB-affected citrus by trunk injection, while the CLas titer was decreased, the abundance of Actinomycetales, Frankiaceae, and Microbacteriaceae was reduced and that of Verrucomicrobia and Bacilli was enhanced [13]. Therefore, the endophytic microbial communities were associated with the progression of citrus HLB following oxytetracycline and penicillin treatment.

We hypothesize that penicillin displayed greater antibacterial activity against CLas than oxytetracycline, which may be associated with the alteration of the structure and function of endophytic microbial communities in HLB-affected citrus in response to these antibiotics. Therefore, in this study, oxytetracycline or penicillin was applied to HLB-affected citrus by a foliar spray. Tissues from the treated trees were subjected to shotgun metagenomic analysis to reveal the mechanism of penicillin and oxytetracycline against CLas.

\section{Materials and Methods}

\subsection{Plant Materials}

Two-year-old healthy grapefruit (Citrus paradisi) seedlings were graft-inoculated with HLB-affected lemon (C. limon) scions and subsequently maintained in the greenhouse. After 10 months, typical HLB symptoms such as vein corking and blotchy mottles appeared on the leaves of the inoculated seedlings. HLB-affected citrus seedlings with typical HLB symptoms were then tested for the presence of CLas bacteria using quantitative real-time polymerase chain reaction (qPCR) with CLas-specific primers (HLBas, HLBr, and HLBp) [14]. 


\subsection{Application of Antibiotics to HLB-Affected Citrus}

Oxytetracycline hydrochloride (Oxy) (Aladdin Industrial Corporation, Shanghai, China) and penicillin G sodium (Pen) (Aladdin Industrial Corporation, Shanghai, China) were prepared as $500 \mathrm{mg} / \mathrm{L}$ chemical solution, respectively. Tap water was selected as control (CK). One liter of the prepared chemical solution (Oxy, Pen, and CK) was applied to 2 year-old HLB-affected grapefruit seedlings that exhibited typical HLB symptoms by foliar spray four times at 2-week intervals. Three biological replicates were performed for each treatment. The treated seedlings were grown at $28^{\circ} \mathrm{C} \pm 5{ }^{\circ} \mathrm{C}$ in an insect-proof greenhouse. Five leaf samples were collected from each treatment at 0 and 90 days after initial treatment (DAT), and DNA was extracted for qPCR and metagenomic analysis.

\subsection{Graft-Based Assay}

A graft-based assay was used to evaluate the effectiveness of avermectin, which is produced from S. avermitilis, against CLas. HLB-infected budsticks were collected from symptomatic rough lemons, and confirmed to be positive for CLas by real-time qPCR [4,14]. The budsticks (20 scions per treatment per concentration) were soaked in $500 \mathrm{mg} / \mathrm{L}$ avermectin solution overnight in a fume hood under ventilation and continuous fluorescent light. Each soaked budstick was cut into a 2-bud scion and grafted onto individual 2-year-old HLB-free grapefruit (Citrus paradisi "Duncan") rootstock seedlings and the grafts were covered with plastic tape for three weeks. To enhance scion growth, new flush from the rootstocks was removed immediately after grafting. Grafted plants were shaded and maintained at $25^{\circ} \mathrm{C} \pm 2{ }^{\circ} \mathrm{C}$ in an insect-proof greenhouse. The effectiveness of the chemical treatments against $\mathrm{CLas}$ was determined by measuring the titer of CLas in both the grafted scion and the rootstock using qPCR. Briefly, five leaves were randomly sampled from the scion (rough lemon) and rootstock (grapefruit) 180 days after grafting. The leaves were washed in tap water and then rinsed three times with sterile water. Midribs were excised, frozen in liquid nitrogen, and stored at $-80^{\circ} \mathrm{C}$ for further processing. The midribs of five leaves from each sample were pooled and used for DNA extraction and subsequent qPCR analysis as described [4,14].

\subsection{Genomic DNA Extraction and $q P C R$ Analysis}

Each leaf was rinsed three times with sterile water. Midribs were separated from the leaf samples and cut into pieces 1.0 to $2.0 \mathrm{~mm}$ in length. DNA was extracted from $0.1 \mathrm{~g}$ (fresh weight) of leaf midrib tissue using a DNeasy Plant Mini Kit (Qiagen, Valencia, CA, USA) according to the manufacturer's protocol. qPCR was performed with primers and probes (HLBas, HLBr and HLBp) for CLas [14] using the ABI 7500 Fast Real-Time PCR System (Applied Biosystems, Foster City, CA, USA) in a $20 \mu \mathrm{L}$ reaction volume containing $300 \mathrm{nM}$ (each) target primer (HLBas and $\mathrm{HLBr}$ ), $150 \mathrm{nM}$ target probe (HLBp), and $1 \times$ TaqMan qPCR Mix (Applied Biosystems). The amplification protocol was $95{ }^{\circ} \mathrm{C}$ for $20 \mathrm{~s}$ followed by 40 cycles at $95^{\circ} \mathrm{C}$ for $3 \mathrm{~s}$ and $60^{\circ} \mathrm{C}$ for $30 \mathrm{~s}$. All reactions were performed in triplicate and each run contained one negative (DNA from healthy plant) and one positive (DNA from a CLas-infected plant) control. The positive control was from the same sample, and was checked to ensure that the Ct remained constant. Data were analyzed using the ABI 7500 Fast Real-Time PCR System with SDS software.

\subsection{DNA Extraction, Library Preparation, and Sequencing for Metagenomic Analysis}

For shortgun metagenomic analysis, the leaf samples were chemically sterilized with $\sim 5 \%$ sodium hypochlorite for $2 \mathrm{~min}$ to remove phyllosphere bacteria. DNA was extracted 90 days after the initial chemical treatment as described above. DNA from five leaves in each treatment was pooled in equal amounts from the three replicates. Afterward, the DNA was sheared into fragments of approximately 300 bp using an M220 Focused-ultrasonicator (Covaris Inc., Woburn, MA, USA) to build a paired-end library. DNA templates were then pretreated using a Tru-seq Kit according to the manufacturer's instructions (https://www.illumina.com/). The libraries were pooled and loaded onto an Illumina 
cBot [15]. Pair-end sequencing $(2 \times 150 \mathrm{bp})$ was performed on a Hiseq2000 sequencer (Illumina) according to the standard protocol (https://www.illumina.com/).

\subsection{Bioinformatics Analyses}

Raw reads from metagenome sequencing were filtered, trimmed, and quality-controlled to generate clean reads, which were further trimmed using Sickle with the parameters $-\mathrm{q} 20$ and -180 [16]. The trimmed reads were aligned to the sweet orange genome [17] using Bowtie [18] and the corresponding mapped reads were removed. Only reads that did not map to the citrus genome were retained for further analysis.

Metagenome taxonomy was assigned using small-subunit (SSU) rRNA gene tags and predicted proteins [19]. The SSU rRNA gene tags were obtained by aligning the raw reads with the Silva SSU rRNA database (http://www.arb-silva.de/) using an optimized E-value threshold of $1 \times 10^{-5}$, an alignment length of $100 \mathrm{bp}$, and an identification cutoff of $80 \%$. The taxonomic annotation of the samples was obtained by BLAST against the NCBI-nt database. For each protein-coding read, alignment with protein sequences in the NCBI-nr database was performed using the BLAST tool, and results with the best hit to the NCBI microbial taxonomy database were obtained.

The network of association between bacterial species within CK-, Oxy-, and Pen-treated samples was generated with SparCC methods [20]. The Shannon and Simpson biodiversity indices were calculated as:

$$
\text { Shannon's index }=-\sum_{1}^{\mathrm{n}} F \mathrm{i} L n F i \text { and Simpson's diversity index }=1-\sum_{1}^{\mathrm{n}} \mathrm{Fi}^{2}
$$

where $\mathrm{n}$ represents the richness or total number of families, $F i$ is the relative species abundance, $i^{\text {th }}$ is the number of species was detected, and $L n$ is the natural logarithm.

Metagenes were predicted by MetaGeneMark. Non-redundant gene categories (uni-genes) were generated using CD-HIT-est with an identity cutoff of 95\% [21]. To obtain taxonomic annotation for the unigenes, the protein sequences were aligned against the NCBI microbial NR database using DIAMOND software [22] with an E value cutoff of $1 \times 10^{-5}$. Then, analysis of taxonomic and functional abundance was conducted by Kraken (0.10.5-beta) [23] with a custom database. Functional annotation was assigned to the unigenes by BLASTing against the NR, COG, KEGG, GO, Pfam, and Swissprot databases using DIAMOND software. The Venn diagram, heatmap and correlation analysis were performed using R packages (v3.2.0).

\subsection{Statistical Analyses}

Variance analysis was conducted to analyze the antibacterial activity of antibiotics. The data of antibiotics treatments were analyzed by Duncan's multiple range test at $p<0.05$. All the data analysis was run in SAS software package (SAS V.9.1, SAS institute, Cary, NC, USA).

\section{Results}

\subsection{Efficacy of Oxy and Pen against CLas}

The antibiotics were applied to HLB-affected citrus as foliar sprays in greenhouse. Ninety days after the initial treatment, the CLas titer in the HLB-affected citrus was significantly reduced by both Oxy $(p=0.0007)$ and Pen $(p=0.0020)$ (Figure 1). For CK treatment, the CLas titer was not significantly changed $(p=0.1099)$ and remained at high levels (Figure 1). Pen (Ct value $=36.38 \pm 2.33$ ) treatment had significantly greater antibacterial activity against CLas compared to Oxy treatment $(C t$ value $=29.76 \pm 1.45)(p=0.0006)$. 


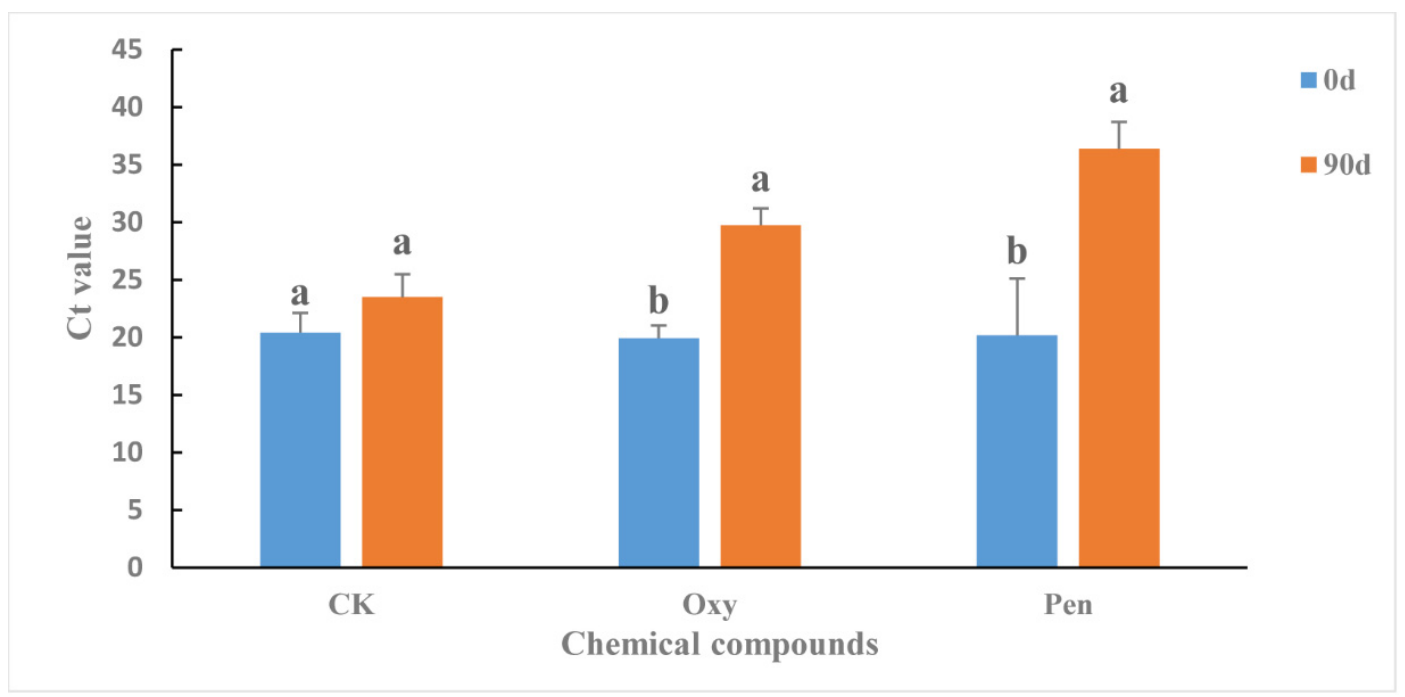

Figure 1. Ct value in HLB-affected citrus treated with oxytetracycline (Oxy) and penicillin (Pen). Different letters represented significantly differences at the level of $0.05(p \leq 0.05)$.

3.2. Structure and Function of the Endophytic Microbial Community in HLB-Affected Citrus Leaves Treated with Oxy and Pen

Metagenomic analysis of leaf samples collected from HLB-affected citrus treated with Oxy, Pen, or CK yielded 2,254,884, 3,448,840, and 2,453,023 clean reads, respectively (Table 1). Proteobacteria was the most abundant phylum in all three treatments (Figure 2A). Cyanobacteria and Actinobacteria were abundant in plants treated with Oxy and Pen, respectively (Figure 2A). For CK-treated plants, Candidatus Liberibacter asiaticus species was more abundant than in Oxy- and Pen-treated plants. The relative abundance of Candidatus Liberibacter asiaticus following Oxy treatment was much higher than that seen for Pen treatment (Figure 2B). The relative abundance of Streptomyces avermitilis species was much higher after Oxy and Pen treatment, relative to that seen for CK (Figure 2B). In particular, the highest relative abundance of this species was seen in Pen-treated plants. In total, we identified 459 bacterial species, of which 151 were present in all three treatments (Figure 2C). CK had the highest number of species (327), while there were 226 and 208 species detected in Oxy- and Pen-treated plants, respectively (Table S1). Moreover, Shannon's and Simpson's diversity indices both revealed that Oxy-treated plants had higher species diversity compared to Pen-treated plants (Figure 2D).

Table 1. Summary of metagenome data.

\begin{tabular}{ccccccc}
\hline Treatment & Raw Data (bp) & Clean Data (bp) & Clean Reads & GC (\%) & Q20 (\%) & Q30 (\%) \\
\hline CK & $794,635,500$ & $676,465,200$ & $2,254,884$ & 42.81 & 93.88 & 87.72 \\
Oxy & $1,228,026,300$ & $1,034,652,000$ & $3,448,840$ & 42.13 & 94.84 & 89.12 \\
Pen & $853,398,900$ & $735,906,900$ & $2,453,023$ & 40.74 & 94.39 & 88.5 \\
\hline
\end{tabular}

Under the antibiotics treatment, the correlation of CLas between the other 458 bacterial species was analyzed (Table S1). The results indicated that 11 bacterial species, relative abundance of which was more than 0.0001 , had significantly correlated with CLas $(1>|\mathrm{R}|>0.9, p<0.05)$. The bacterial species including Propionibacterium acnes, Pseudomonas putida, Synechocystis sp_PCC_6803, Sphingomonas wittichii, Staphylococcus epidermidis, and Deinococcus gobiensis, had positively correlated with CLas (Figure 3). On the other hand, five bacterial species such as Streptomyces avermitilis, Alteromonas macleodii, Bradyrhizobium japonicum, Bradyrhizobium sp S23321, and Bradyrhizobium oligotrophicum, showed negative correlation with CLas (Figure 3). 

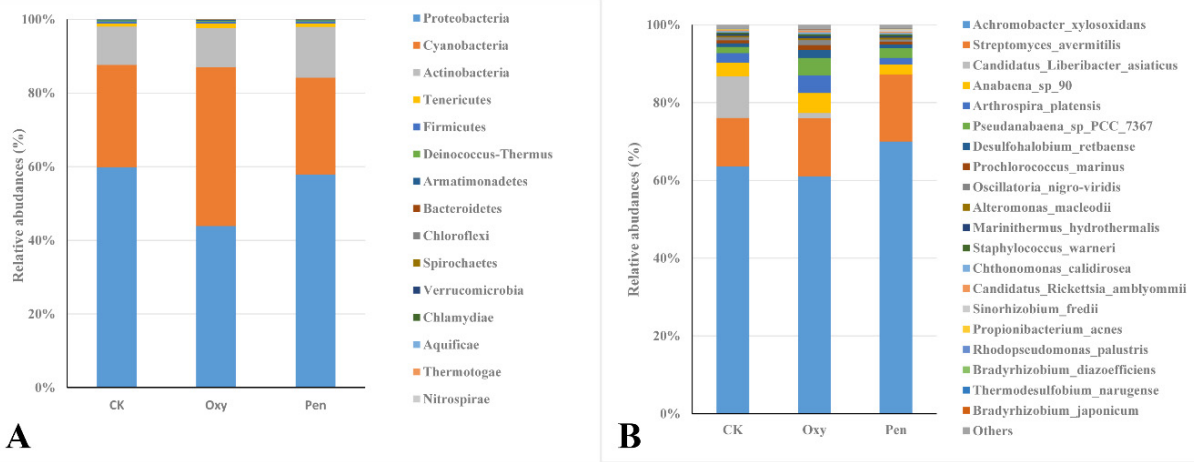

C
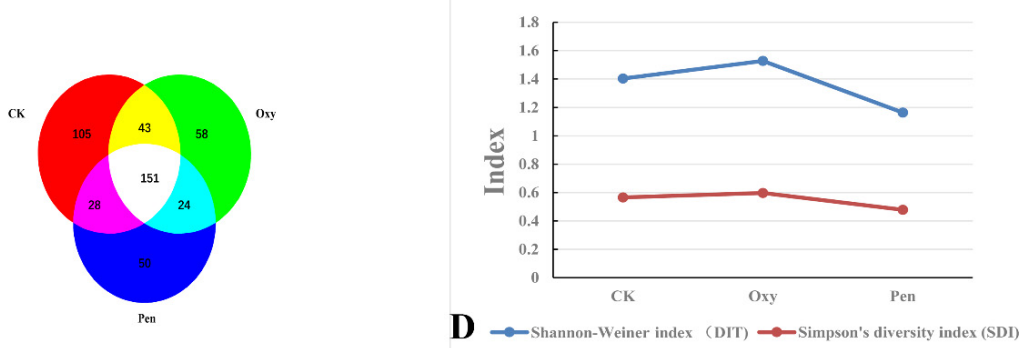

Figure 2. Bacterial species present after antibiotic treatments of HLB-affected citrus plants. Taxonomic abundance of microbial community at the (A) phyla and (B) species rank in HLB-affected citrus treated with oxytetracycline (Oxy) or penicillin (Pen). (C) Venn diagram representing bacterial species in the endophytic microbial community in HLB-affected citrus treated with tap water (CK), oxytetracycline (Oxy), or penicillin (Pen). (D). Simpson's diversity index (SDI) and Shannon-Weiner index (DIT) of the endophytic microbial community in HLB-affected citrus treated with tap water (CK), oxytetracycline (Oxy), or penicillin (Pen).

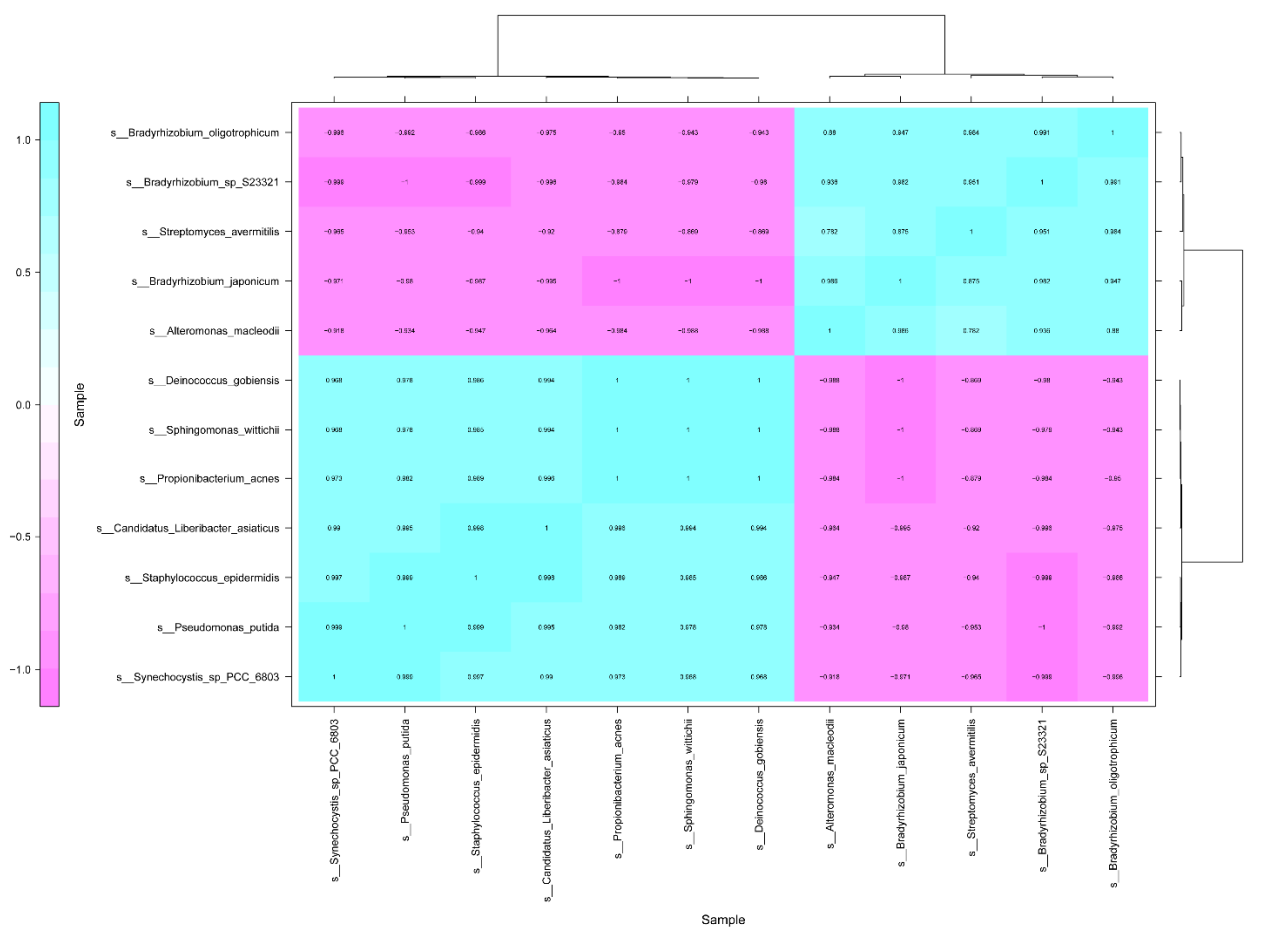

Figure 3. Correlation analysis of Candidatus Liberibacter asiaticus between other bacterial species in HLB-affected citrus leaves in response to the antibiotics. 
Overall, 3380 unigenes were annotated against at least one of the public databases (Table 2). There were 1351 genes annotated against the COG database (Table 2). The relative abundance of energy production and conversion $(\mathrm{C})$ and transcription $(\mathrm{K})$ were enhanced by Oxy and Pen treatments (Figure 4A). In particular, the relative abundance of cell motility was lowest for Pen-treated plants.

Table 2. Number of unigenes annotated against COG, GO, KEGG, Nt, Pfam, and Swissprot databases.

\begin{tabular}{cccc}
\hline Anno_Database & Annotated_Number & $\mathbf{3 0 0} \leq$ Length $<\mathbf{1 0 0 0}$ & Length $\geq \mathbf{1 0 0 0}$ \\
\hline COG_Annotated & 1351 & 146 & 2 \\
GO_Annotated & 1296 & 133 & 1 \\
KEGG_Annotated & 1796 & 147 & 2 \\
Nt_Annotated & 2145 & 216 & 5 \\
Pfam_Annotated & 2222 & 231 & 4 \\
Swissprot_Annotated & 2548 & 223 & 4 \\
All_Annotated & 3380 & 242 & 5 \\
\hline
\end{tabular}
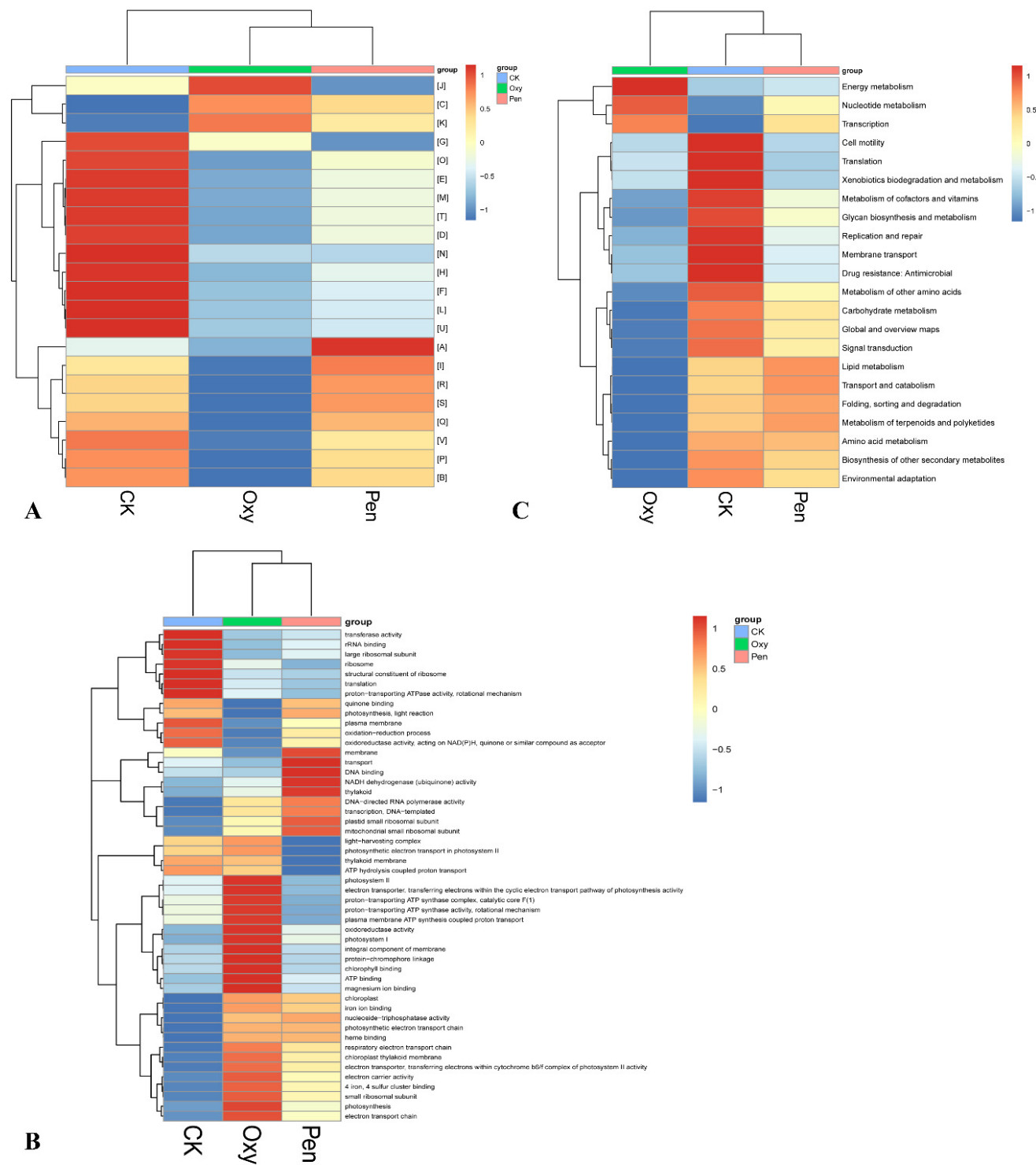

Figure 4. Heatmap of COG (A), GO (B), and KEGG (C) function comparison of the microbiome in HLB-affected citrus in response to oxytetracycline (Oxy) and penicillin (Pen) treatment. 
In total, 1296 genes were annotated against the GO database (Table 2). The relative abundance of chloroplast, chloroplast thylakoid membrane, photosynthesis, nucleoside-triphosphatase activity, iron ion binding, 4 iron, 4 sulfur cluster binding, electron carrier activity, electron transport chain, photosynthetic electron transport chain, heme binding, respiratory electron transport chain, electron transporter, transferring electrons within cytochrome b6/f complex of photosystem II activity, and small ribosomal subunit were increased by both Oxy and Pen treatment (Figure 4B). In the Pen treatment, the relative abundance of membrane, NADH dehydrogenase (ubiquinone) activity, transport, thylakoid, DNA binding, DNA-directed RNA polymerase activity, plastid small ribosomal subunit, mitochondrial small ribosomal subunit, and transcription, DNA-templated were the highest relative to that for CK and Oxy (Figure 4B).

For KEGG annotation, 1796 genes were searched against the KEGG database (Table 2). The relative abundance of energy metabolism, nucleotide metabolism, and transcription were increased by both Oxy and Pen (Figure 4C). Notably, there were no unigenes detected for cell motility pathways in response to Pen.

\subsection{Effect of Avermectin Streptomyces avermitilis on Candidatus Liberibacter asiaticus}

Streptomyces avermitilis can produce avermectin. Therefore, the effectiveness of avermectin in reducing CLas titer was also evaluated by grafted-based assay. Although the CLas titer was not significantly different between avermectin- and tap water-treated (CK) scions (Figure 5), in rootstocks the CLas titer was much lower for the avermectin-treated plants compared to the CK plants $(p=0.0447)$.

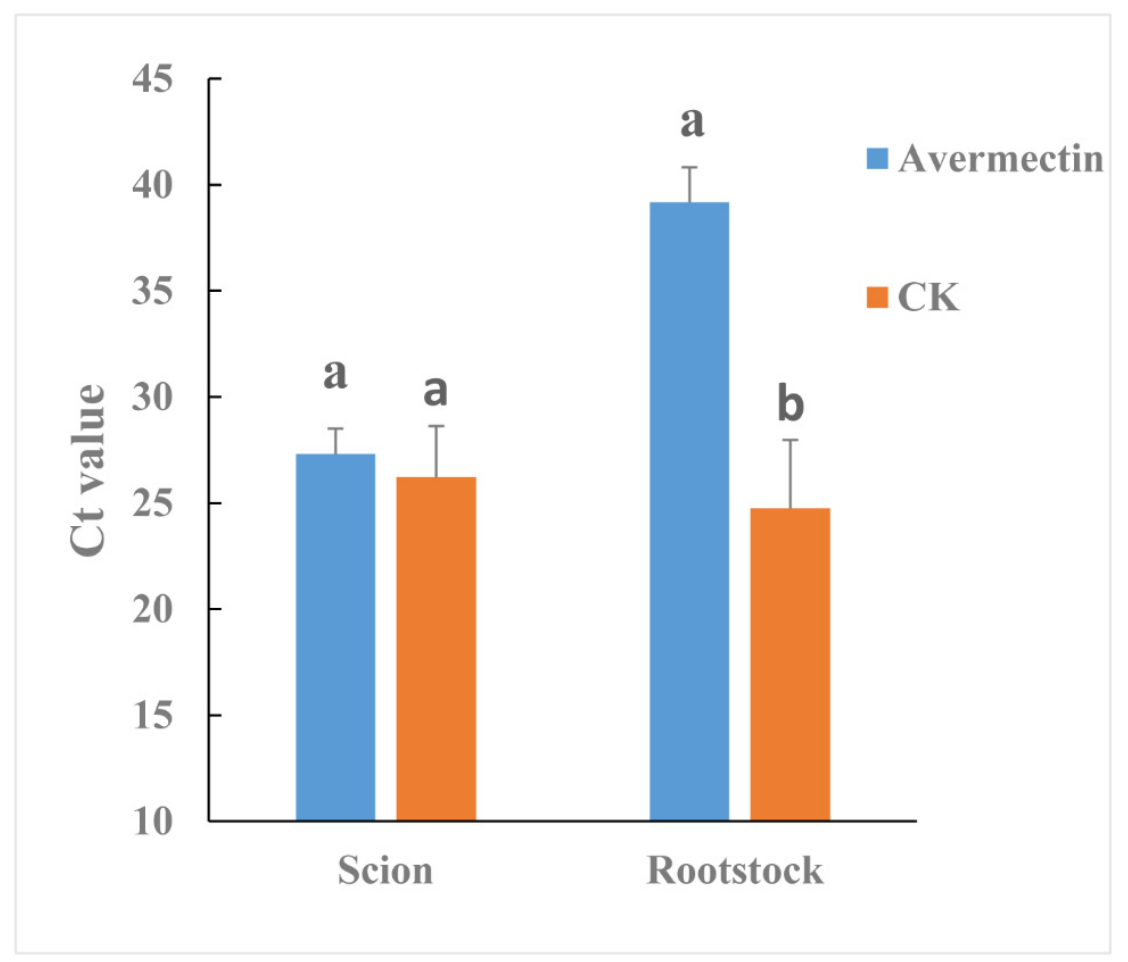

Figure 5. Effectiveness of avermectins against CLas as evaluated by a grafted-based assay. Different letters represented significantly differences at the level of $0.05(p \leq 0.05)$.

\section{Discussion}

In this study, Pen displayed greater antibacterial activity against CLas than did Oxy (Figure 1). In addition, antibiotic treatment could change the microbial structure and function in HLB-affected citrus. Therefore, the alteration of CLas-associated microbiota may have contributed to the greater antibacterial activity of Pen. 
Endophyte microorganisms can control plant pathogens [24] and enhance plant growth [25] through endophyte-mediated synthesis of natural compounds and metabolites. Our research indicated that $S$. avermitilis species were enriched in HLB-affected citrus following antibiotic treatment, especially relative abundance of this bacterial species was much higher in Pen treatment, relative to Oxy treatment (Figure 2B). Correlation analysis also indicated that this species may inhibit $C$ Las growth $(R=-0.92)$ (Figure 3). Streptomyces. avermitilis can produce avermectins [26], which have activity against helminths, insects, and arachnids [26,27]. Avermectins also have antibacterial activity against mycobacterial species [28]. The higher relative abundance induced by Pen treatment, may enhance avermectin production by $S$. avermitilis. In addition, the graft-based assay indicated that avermectins did not eliminate CLas, but did inhibit CLas transmission from scion to rootstock (Figure 5). Unigenes associated with cell motility were absent in Pen-treated tissues (Figure 4A), which may indicate that avermectin production by $S$. avermitilis induced by Pen treatment could inhibit mobility of endophytic bacteria, especially $C$ Las. Therefore, the enrichment of $S$. avermitilis under Pen treatment, may enhance antibacterial activity against $C$ Las (Figure 6) by inhibiting the mobility.

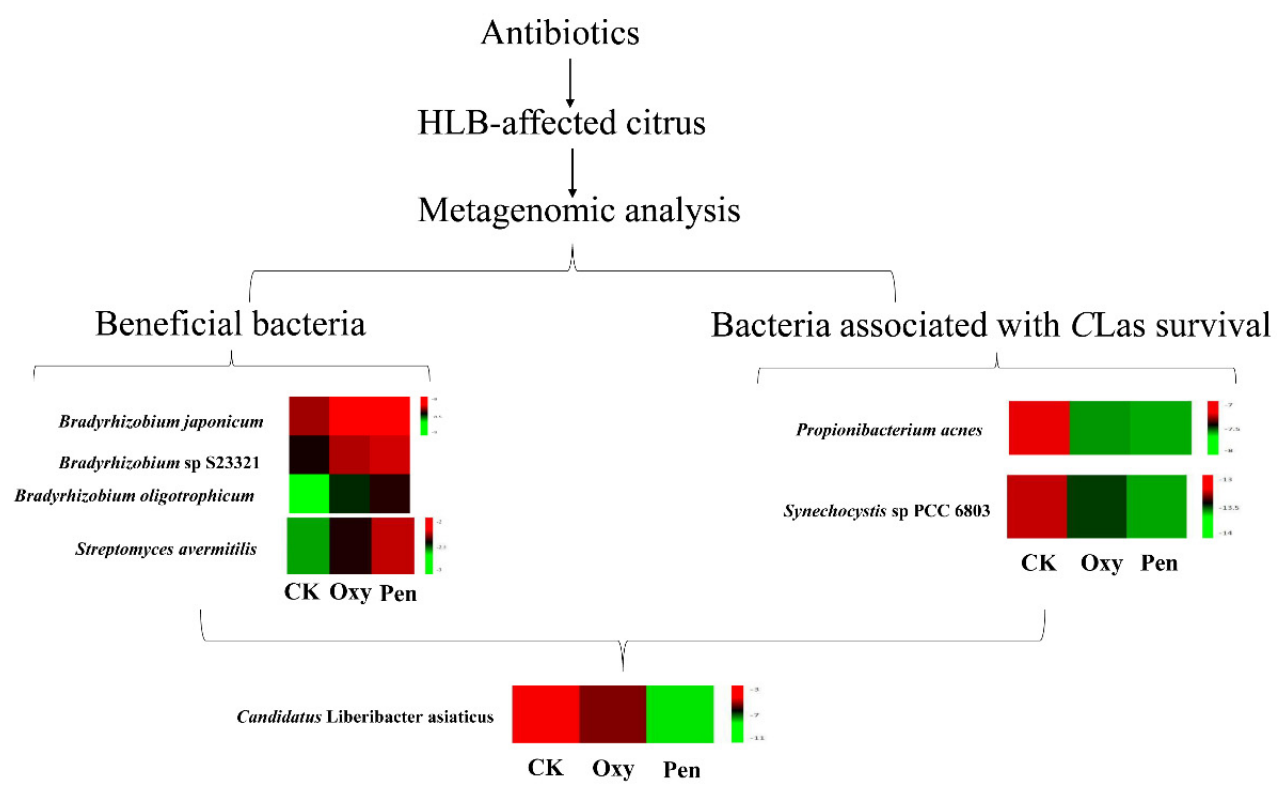

Figure 6. The mechanism of the antibiotics (oxytetracycline (Oxy) and penicillin (Pen)) against Candidatus Liberibacter asiaticus (CLas) based on metagenomic analysis.

Bradyrhizobium sp. is a slow-growing nitrogen-fixing symbiotic bacterium of legumes and common root endophyte of other plants. This bacterium, which is considered to be a plant growth-promoting rhizobacterium (PGPR), is capable of colonizing the roots of non-legumes and produce phytohormones and siderophores as well as exhibit antagonistic effects toward several plant pathogens [29]. A recent study also demonstrated that CLas can decrease the relative abundance of most rhizoplane-enriched genera, such as Bradyrhizobium, and reduce the relative abundance of the functional attributes involved in microbe-plant interactions [30]. Therefore, Bradyrhizobium is beneficial for citrus health. In this study, the relative abundance of three Bradyrhizobium species was highest in the Pen treatment group compared with Oxy and CK treatment groups (Figure 6). Moreover, a previous study indicated that unigenes in Bradyrhizobium in healthy citrus rhizoplane-upregulated was enriched in the categories of "transcription" and "metabolism|unclassified" compared to HLB-affected citrus [30]. Our study also showed that the relative abundance of several GO terms, including DNA binding, DNA-directed RNA polymerase activity and transcription, and DNA-templated, which were associated with transcription, were enhanced by the antibiotics and the relative abundance in the Pen treatment group was much higher than in the Oxy treatment group (Figure 4B). Therefore, enhancement of the relative abundance 
of transcription by enriched Bradyrhizobium may contribute to the greater antibacterial activity of Pen against CLas (Figure 6).

Results from an earlier study suggested that CLas could use ecological services derived from CLas-associated microbiota to colonize the host and generate pathogen-associated community that stimulates disease development [12]. Our study found that several bacterial species including Propionibacterium acnes, Pseudomonas putida, Synechocystis sp PCC 6803, and Staphylococcus epidermidis, have a positive association with CLas. The relative abundance of these bacteria was decreased by both antibiotics, and the relative abundance were much lower in Pen treatment, relative to that in Oxy treatment (Figure 3).

Propionibacterium acnes as Gram-positive bacterium, belonged to Actinobacteria. In previous study, although CLas was not isolated in axenic culture, it was isolated in co-cultures with actinobacteria closely related to $P$. acnes. The co-cultures remained after attempts to purify the cultures by single-colony isolation, suggesting that the bacteria might be mutually beneficial to each other in culture [31]. In addition, the Znu system, a member of $A B C$ transporter family, is critical for survival and pathogenesis of CLas [32]. Previous finding indicated a role in zinc uptake for the ZnuA, ZnuB, and ZnuC proteins from Sinorhizobium meliloti as well as one homologous gene cluster in CLas, suggesting this system was associated with growth and pathogenesis of CLas [33]. The sequence alignment of CLas-ZnuA2 sequence showed 24\% identity with Synechocystis [34]. In this paper, the bacterial species Synechocystis sp PCC 6803 may share functional znuA homologues that encode for a high affinity zinc uptake system associated with growth and virulence of CLas. Therefore, the lower relative abundance of bacteria species associated with CLas survival following Pen treatment may have resulted in greater antibacterial activity against $C$ Las.

However, it was found that Pseudomonas putida could significantly reduce the population of viable CLas in HLB symptomatic leaves [35]. In this study, relative abundance of CLas in HLB-affected citrus leaves was decreased by the antibiotics, as well as that of Pseudomonas putida (Table S1). Meanwhile, there are still no reports about the function of Staphylococcus epidermidis in citrus. Therefore, whether Pseudomonas putida and Staphylococcus epidermidis are associated with CLas survival is still unknown. It will be studied in further research.

\section{Conclusions}

Although it is difficult to elucidate the mechanism of action of an antibiotic against uncultured bacteria CLas, metagenomic analysis can provide new insight into the endophytic microbial community in HLB-affected citrus following antibiotic treatment, which was associated with antibacterial activity of the antibiotic against CLas. Our study indicated that the relative abundance of beneficial bacterial species, including S. avermitilis and Bradyrhizobium, was higher in Pen-treated plants compared to those treated with Oxy, and the abundance of the bacterial species (such as Propionibacterium acnes and Synechocystis sp PCC 6803) associated with CLas survival was lower for Pen-treated plants compared to Oxy-treated plants, indicating that Pen has greater antibacterial activity against CLas. Moreover, the beneficial bacteria in this study will be a potential candidate for biocontrol of citrus HLB. Now, isolation and identification of these two beneficial bacteria are undergoing. Propionibacterium acnes and Synechocystis sp PCC 6803 can be selected for target bacteria to decrease CLas survival. Therefore, this study not only developed a novel strategy for studying modes of action of antimicrobials against uncultured bacteria, but also provided valuable insight for developing eco-friendly and effective strategies to combat citrus HLB or other plant bacteria pathogen.

Supplementary Materials: The following are available online at http://www.mdpi.com/2079-6382/9/12/874/s1. Table S1. Abundance of bacterial species in endophytic microbial communities in HLB-affected citrus treated with tap water (CK), oxytetracycline (OXY) or penicillin (Pen). Sequencing data. All the supplementary material can be also found: https://zenodo.org/record/3612711\#.XiTXTNIzbcc. 
Author Contributions: Conceived and designed the experiment: C.Y., Y.D., and M.Z. Performed the experiments: C.Y., H.H., Y.W., X.L. Analyzed the data: C.Y., M.Z. Contributed reagents/materials/analysis tools: G.F., C.P., Y.D. Wrote and revised the paper: C.Y., M.Z., V.A., C.P., and Y.D. All authors have read and agreed to the published version of the manuscript.

Funding: This work is funded by Fujian Natural Science Foundation. (Project 2018J05050), Fujian Special Fund for Scientific Research Institutes in the Public Interest. (Project 2018R1013-6), Science and Technology Major Project of Guangxi (Guike AA 18118046).

Acknowledgments: We greatly appreciate Bioscience Editing Solutions for critically reading this paper and providing helpful suggestions.

Conflicts of Interest: The authors declare no conflict of interest.

Data Availability Statement: The data that support the findings of this study are available from the corresponding author upon reasonable request.

\section{References}

1. Bové, J.M. Huanglongbing: A destructive, newly-emerging, century-old disease of citrus. J. Plant Pathol. 2006, 88, 7-37.

2. Gottwald, T.R. Current epidemiological understanding of citrus Huanglongbing. Annu. Rev. Phytopathol. 2010, 48, 119-139. [CrossRef] [PubMed]

3. Blaustein, R.A.; Lorca, G.L.; Teplitski, M. Challenges for managing Candidatus Liberibacter spp. (Huanglongbing disease pathogen): Current control measures and future directions. Phytopathology 2018, 108, 424-435. [CrossRef] [PubMed]

4. Zhang, M.; Guo, Y.; Powell, C.A.; Doud, M.S.; Yang, C.; Duan, Y. Effective antibiotics against 'Candidatus Liberibacter asiaticus' in HLB-affected citrus plants identified via the graft-based evaluation. PLoS ONE 2014, 9, e111032. [CrossRef] [PubMed]

5. Hu, J.; Wang, N. Evaluation of the Spatiotemporal Dynamics of Oxytetracycline and Its Control Effect against Citrus Huanglongbing via Trunk Injection. Phytopathology 2016, 106, 1495-1503. [CrossRef] [PubMed]

6. Chopra, I.; Roberts, M. Tetracycline antibiotics: Mode of action, applications, molecular biology, and epidemiology of bacterial resistance. Microbiol. Mol. Biol. Rev. 2001, 65, 232-260. [CrossRef]

7. Schwarz, R.; Van Vuuren, S. Decrease in fruit greening of sweet orange by trunk injection of tetracyclines. Plant. Dis. Rep. 1971, 57, 634-637.

8. Spratt, B.G.; Cromie, K.D. Penicillin-binding proteins of gram-negative bacteria. Clin. Infect. Dis. 1988, 10, 699-711. [CrossRef]

9. Pankey, G.; Sabath, L. Clinical relevance of bacteriostatic versus bactericidal mechanisms of action in the treatment of Gram-positive bacterial infections. Clin. Infect. Dis. 2004, 38, 864-870. [CrossRef]

10. Yang, C.; Powell, C.A.; Duan, Y.; Shatters, R.; Zhang, M. Antimicrobial nanoemulsion formulation with improved penetration of foliar spray through citrus leaf cuticles to control citrus huanglongbing. PLoS ONE 2015, 10, e0133826. [CrossRef]

11. Brader, G.; Compant, S.; Vescio, K.; Mitter, B.; Trognitz, F.; Ma, L.-J.; Sessitsch, A. Ecology and genomic insights into plant-pathogenic and plant-nonpathogenic endophytes. Annu. Rev. Phytopathol. 2017, 55, 61-83. [CrossRef]

12. Fujiwara, K.; Iwanami, T.; Takashi, F. Alterations of Candidatus Liberibacter asiaticus-associated microbiota decrease survival of Ca. L. asiaticus in in vitro assays. Front. Microbiol. 2018, 9, 3089. [CrossRef]

13. Ascunce, M.S.; Shin, K.; Huguet-Tapia, J.C.; Poudel, R.; Garrett, K.A.; van Bruggen, A.H.; Goss, E.M. Penicillin trunk injection affects bacterial community structure in citrus trees. Microb. Ecol. 2019, 78, 457-469. [CrossRef] [PubMed]

14. Li, W.; Hartung, J.S.; Levy, L. Quantitative real-time PCR for detection and identification of Candidatus Liberibacter species associated with citrus huanglongbing. J. Microbiol. Methods 2006, 66, 104-115. [CrossRef] [PubMed]

15. Clark, M.J.; Chen, R.; Lam, H.Y.; Karczewski, K.J.; Chen, R.; Euskirchen, G.; Butte, A.J.; Snyder, M. Performance comparison of exome DNA sequencing technologies. Nat. Biotechnol. 2011, 29, 908. [CrossRef] [PubMed]

16. Joshi, N.; Fass, J. Sickle: A Sliding-Window, Adaptive, Quality-Based Trimming Tool for FastQ Files; Version 1.33; [Software]; GitHub: San Francisco, CA, USA, 2011. 
17. Xu, Q.; Chen, L.-L.; Ruan, X.; Chen, D.; Zhu, A.; Chen, C.; Bertrand, D.; Jiao, W.-B.; Hao, B.-H.; Lyon, M.P. The draft genome of sweet orange (Citrus sinensis). Nat. Genet. 2013, 45, 59. [CrossRef]

18. Langmead, B.; Salzberg, S.L. Fast gapped-read alignment with Bowtie 2. Nat. Meth. 2012, 9, 357. [CrossRef]

19. Parks, D.H.; Beiko, R.G. Identifying biologically relevant differences between metagenomic communities. Bioinformatics 2010, 26, 715-721. [CrossRef]

20. Friedman, J.; Alm, E.J. Inferring correlation networks from genomic survey data. PLoS Comput. Biol. 2012, 8, e1002687. [CrossRef]

21. Fu, L.; Niu, B.; Zhu, Z.; Wu, S.; Li, W. CD-HIT: Accelerated for clustering the next-generation sequencing data. Bioinformatics 2012, 28, 3150-3152. [CrossRef]

22. Buchfink, B.; Xie, C.; Huson, D.H. Fast and sensitive protein alignment using DIAMOND. Nat. Meth. 2015, 12, 59. [CrossRef] [PubMed]

23. Wood, D.E.; Salzberg, S.L. Kraken: Ultrafast metagenomic sequence classification using exact alignments. Genome Biol. 2014, 15, R46. [CrossRef] [PubMed]

24. Sturz, A.; Matheson, B. Populations of endophytic bacteria which influence host-resistance to Erwinia-induced bacterial soft rot in potato tubers. Plant Soil 1996, 184, 265-271. [CrossRef]

25. Bent, E.; Chanway, C.P. The growth-promoting effects of a bacterial endophyte on lodgepole pine are partially inhibited by the presence of other rhizobacteria. Can. J. Microbiol. 1998, 44, 980-988. [CrossRef]

26. Burg, R.W.; Miller, B.M.; Baker, E.E.; Birnbaum, J.; Currie, S.A.; Hartman, R.; Kong, Y.-L.; Monaghan, R.L.; Olson, G.; Putter, I. Avermectins, new family of potent anthelmintic agents: Producing organism and fermentation. Antimicrob. Agents Chemother. 1979, 15, 361-367. [CrossRef] [PubMed]

27. Hotson, I. The avermectins: A new family of antiparasitic agents. J. S. Afr. Vet. Assoc. 1982, 53, 87-90. [PubMed]

28. Lim, L.E.; Vilchèze, C.; Ng, C.; Jacobs, W.R.; Ramón-García, S.; Thompson, C.J. Anthelmintic avermectins kill Mycobacterium tuberculosis, including multidrug-resistant clinical strains. Antimicrob. Agents Chemother. 2013, 57, 1040-1046. [CrossRef]

29. Antoun, H.; Beauchamp, C.J.; Goussard, N.; Chabot, R.; Lalande, R. Potential of Rhizobium and Bradyrhizobium species as plant growth promoting rhizobacteria on non-legumes: Effect on radishes (Raphanus sativus L.). In Molecular Microbial Ecology of the Soil; Springer: Berlin, Germany, 1998; pp. 57-67.

30. Zhang, Y.; Xu, J.; Riera, N.; Jin, T.; Li, J.; Wang, N. Huanglongbing impairs the rhizosphere-to-rhizoplane enrichment process of the citrus root-associated microbiome. Microbiome 2017, 5, 97. [CrossRef]

31. Davis, M.J.; Mondal, S.N.; Chen, H.; Rogers, M.E.; Brlansky, R.H. Co-cultivation of 'Candidatus Liberibacter asiaticus' with actinobacteria from citrus with huanglongbing. Plant. Dis. 2008, 92, 1547-1550. [CrossRef]

32. Duan, Y.; Zhou, L.; Hall, D.G.; Li, W.; Doddapaneni, H.; Lin, H.; Liu, L.; Vahling, C.M.; Gabriel, D.W.; Williams, K.P. Complete genome sequence of citrus huanglongbing bacterium,'Candidatus Liberibacter asiaticus' obtained through metagenomics. Mol. Plant. Microbe Interact. 2009, 22, 1011-1020. [CrossRef]

33. Vahling-Armstrong, C.M.; Zhou, H.; Benyon, L.; Morgan, J.K.; Duan, Y. Two plant bacteria, S. meliloti and Ca. Liberibacter asiaticus, share functional znuABC homologues that encode for a high affinity zinc uptake system. PLoS ONE 2012, 7, e37340. [CrossRef] [PubMed]

34. Sharma, N.; Selvakumar, P.; Bhose, S.; Ghosh, D.K.; Kumar, P.; Sharma, A.K. Crystal structure of a periplasmic solute binding protein in metal-free, intermediate and metal-bound states from Candidatus Liberibacter asiaticus. J. Struct. Biol. 2015, 189, 184-194. [CrossRef] [PubMed]

35. Trivedi, P.; Spann, T.; Wang, N. Isolation and characterization of beneficial bacteria associated with citrus roots in Florida. Microb. Ecol. 2011, 62, 324-336. [CrossRef] [PubMed]

Publisher's Note: MDPI stays neutral with regard to jurisdictional claims in published maps and institutional affiliations. 